\title{
Geographieunterricht in Schweizer Gymnasien nach der Maturitätsreform - Eine Analyse der neuen Geographielehrpläne
}

\section{Sibylle Reinfried, Zürich}

\section{Einführung}

Bis 1994 gab es in der Schweiz weder landesweit verbindliche noch gesamtschweizerisch standardisierte Bildungsziele. Was gelernt werden musste, um die eidgenössische Maturitätsprüfung ablegen zu können, wurde in den sogenannten «Maturitätsprogrammen» im Anhang zur Verordnung über die eidgenössischen Maturitätsprüfungen (Abb. 1) festgelegt, die neben einigen wenigen Bildungszielen lediglich eine sehr generalisierte Auflistung von Themen, genannt «Stoff», enthalten (SCHWEIZERISCHER BUNDESRAT 1990:14). Diese Verordnung war für alle Maturitätsschulen mit eidgenössischer Anerkennung gültig und diente vielen Schulen mehr als 20 Jahre als einzige Richtlinie für den Unterricht. Oftmals gab es für das Fach Geographie keine weiteren offiziellen curricularen Dokumente, ja es existierte nicht einmal ein Lehrplan. Der Unterricht war lehrerzentriert und stark auf den Erwerb von Fakten ausgerichtet. Da die «Maturitätsprogramme» von Kanton zu Kanton und von Schule zu Schule unterschiedlich interpretiert wurden, besassen Schweizer Maturandinnen und Maturanden grosse Wissensunterschiede und unterschiedliche Fertigkeiten, nicht nur im Fach Geographie, sondern auch in anderen Maturfächern.

\section{Die Schweizerische Maturitätsreform in den 90er Jahren}

Heute besitzt die Schweiz eidgenössisch verbindliche Grundlagen für die gymnasiale Ausbildung auf der Sekundarstufe II, den sogenannten Rahmenlehrplan (SChWEIZERISCHE Konferenz der Kantonalen ERZIEHUNGSDIREKTOREN 1994, CONFÉRENCE SUISSE DES DIRECTEURS CANTONEAUX DE L'INSTRUCTION PUBLIQUe 1994, CONFERENZA SVIZZERA DEI DIRETTORI CANTONALI DELLA PUBBLICA EDUCAZIONE 1994). Er besteht aus allgemeinen Bildungszielen einschliesslich deren Begründungen und Erläuterungen sowie Richtzielen (Abb.2) für jede gymnasiale Disziplin. Für die Geographie beschreiben die Bildungsziele das Schulfach als solches und betonen seine Bedeutung für die (Aus-)Bildung der Gymnasiasten, während die Richtziele in spezifische Grundkenntnisse, Grundfertigkeiten und Grundhaltungen unterteilt sind. Der Rahmenlehrplan sagt allerdings nichts aus über die spezifischen Inhalte, die im Unterricht gelehrt und gelernt werden sollen. Ebensowenig beinhaltet er, was am Ende der gymnasialen Ausbildung im Sinne einer Evaluation erreicht werden soll, und er gibt auch nicht an, wie die Lernfortschritte von Schülerinnen und Schülern evaluiert werden sollen.

Um sicherzustellen, dass der Rahmenlehrplan auch von jenen akzeptiert wird, die später mit ihm arbeiten,

\section{Geographie}

für Typus A, B, C, D, E

\section{Bildungsziel}

Angemessene Kenntnisse der verschiedenen Naturräume der Erde. Verständnis für die Beziehungen zwischen geographischen Gegebenheiten und Zivilisation. Einige Vertrautheit mit den Problemen der Ernährung, der Industrialisierung und der Energieversorgung. Einsicht in die Grenzen der wirtschaftlichen Nutzung und in die Notwendigkeit einer verantwortungsbewussten Gestaltung des Lebensraumes.

\section{Stoff}

- Topographisches Grundwissen über die Schweiz, Europa und die übrigen Erdteile.

- Grundzüge der Geologie der Schweiz.

- Geographische Anschauungs- und Darstellungsmethoden.

- Natur- und Kulturfaktoren.

a. Klima, Gewässer, Bodentypen, Vegetation.

b. Bevölkerung, Siedlung, Wirtschaft, Verkehr.

c. Zusammenwirken dieser Faktoren in typischen Landschaften und Räumen.

- Landschaftswandel, Raumordnung und Umweltschutz.

Abb. 1: Maturitätsprogramm für das Fach Geographie von 1973

Federal guidelines for the Geography curriculum in upper secondary schools, 1973

Programme de maturié de 1973 en géographie

(Schweizerischer BundesRat 1990: 14) 
wurde er gemäss der in der Schweizer Basisdemokratie üblichen politischen Tradition von den Lehrkräften der Sekundarstufe II selber verfasst. Die Initiative zur Reform kam 1987 von der Erziehungsdirektorenkonferenz (EDK), dem bildungspolitischen Gremium, in dem die Erziehungsdirektoren aller Kantone vertreten sind. Für die Geographie erarbeitete in der Folge eine Lehrplangruppe des Vereins Schweizerischer Geographielehrerinnen und -lehrer (VSGg), in dem die meisten Geographielehrkräfte an Schweizer Gymnasien Mitglied sind, eine erste Skizze des Rahmenlehrplans, der dann an alle Mitglieder des Vereins und an alle wichtigen geographischen Institutionen des Landes zur Vernehmlassung ging. Das Dokument wurde auch in verschiedenen Lehrerweiterbildungsveranstaltungen (Kurse der Schweizerischen Zentralstelle für die Weiterbildung von Mittelschullehrpersonen WBZ) und durch die Arbeitsgemeinschaft für Fachdidaktik (AFGg), eine Arbeitsgruppe der WBZ, in der alle Schweizer Geographiedidaktiker auf der Sekundarstufe II vertreten sind, evaluiert, und schliesslich unter Einbezug der verschiedenen Kommentare in seine Entwurfsfassung gebracht (Tschanz 1997: 40). Die Entwürfe aller am Gymnasium unterrichteten Fachdis- ziplinen wurden anschliessend in einem aufwendigen Verfahren validiert und 1994 in Kraft gesetzt. Heute sind für das Fach Geographie drei Versionen gültig, eine für die deutsche Schweiz, eine für die Romandie und eine für das Tessin. Sie stehen stellvertretend für die drei verschiedenen Kulturen. Wie kam es dazu?

Die italienische Fassung des Rahmenlehrplans ist eine Zusammenstellung der deutschen und französischen Version, weil die Tessiner Maturandinnen und Maturanden später mehrheitlich an Hochschulen in den anderen Landesteilen studieren. Die Bildungsziele der italienischen Fassung entsprechen denn auch jenen der deutschen Version, während die Richtziele eher mit jenen der französischen Fassung übereinstimmen. Alle drei Rahmenlehrpläne betonen die Bedeutung der Geographie als Raumwissenschaft. Unterschiedlich ist aber, wie der Schwerpunkt innerhalb der Geographie gesetzt wird. In Anlehnung an die unterschiedlichen Traditionen in den geographischen Wissenschaften in den verschiedenen Kulturen betonen die frankophone und italienische Version die Humangeographie stärker, während die deutsche Fassung mehr Gewicht auf die physische Geographie und die ökologische Perspek-

\section{Der Rahmenlehrplan Geographie für die Maturitätsschulen}

\section{Allgemeine Bildungsziele}

Durch den Geographieunterricht gelangen die Schülerinnen und Schüler zur Einsicht, dass Lebensansprüche, Normen und Haltungen raumprägend sind. Dies soll zu einem verantwortungsbewussten Umgang mit dem Lebensraum führen.

Schülerinnen und Schüler sollen eine Landschaft in ihrer Ganzheit bewusst erleben und sie mit Hilfe geographischer Methoden und Kenntnisse analysieren. Sie sind fähig, sich auf der Erde mit ihren vielfältigen Strukturen zu orientieren. Sie begegnen der Welt, insbesondere anderen Kulturen, mit Offenheit.

Das Zusammenwirken und die gegenseitige Beeinflussung von Mensch und Natur soll verständlich werden. Veränderungen der Lebensräume sind zu erfassen und zu beurteilen.

Die Geographie enthält Elemente des natur- und humanwissenschaftlichen Denkens; deshalb verbindet sie die beiden Bereiche. Sie fördert das Erkennen von Zusammenhängen und regt die fächerübergreifende Behandlung von Themen an.

\section{Richtziele}

\section{Grundkenntnisse}

- Die fachspezifischen Grundbegriffe kennen.

- Über ein topographisches Grundwissen verfügen, um aktuelle Ereignisse geographisch deuten und einordnen zu können.

- Landschaftselemente und ihre raumprägenden Faktoren kennen.

Die Prozesse des Landschaftswandels erfassen.

- In Grundzügen die Geologie der Schweiz kennen. 


\section{Grundfertigkeiten}

- Karten lesen und sich im Gelände zurechtfinden.

- Geographische Darstellungsmethoden anwenden, thematische Karten, Profile, Diagramme, Statistiken, Modelle, Bilder und Texte interpretieren und z.T. selbst entwerfen; Ergebnisse geographischer Untersuchungen verständlich darstellen und weitergeben.

- In Modellen und Fallbeispielen geographische Faktoren erkennen und Prozesse verstehen.

- Landschaftselemente, ihre Wechselwirkungen und Strukturen beobachten, erkennen, interpretieren und beurteilen:

- Ursachen und Zusammenwirken von Naturkräften erkennen.

- Beziehungen zwischen natur- und kulturgeographischen Elementen erfassen.

- Die Wechselwirkungen zwischen den Daseinsfunktionen des Menschen (Wohnen, Arbeiten, Freizeit) und der Umwelt verstehen und beurteilen.

- Erkennen, wie Standortfaktoren die wirtschaftliche Nutzung einer Landschaft bestimmen.

- Bedeutung der gesetzlichen Vorschriften und Folgen ihrer Veränderung für die Landschaft abschätzen.

- Kulturelle und gesellschaftliche Einflüsse auf Raumnutzung und Raumentwicklung erkennen.

- Die zunehmende Verflechtung von Ländern und Kulturen und die daraus resultierenden Veränderungen der Lebensbedingungen einsehen.

\section{Grundhaltungen}

- Die Begegnung mit anderen Menschen, Kulturen und Landschaften als Bereicherung erfahren und durch die Vergleiche die eigene Umwelt besser verstehen.

- Durch persönliche Erlebnisse und Erfahrungen seine Einstellung überdenken, sich auftauchender Probleme bewusst werden und sich für deren Lösung einsetzen.

- Bereit sein, persönliche raumwirksame Tätigkeiten zu hinterfragen und entsprechend verantwortungsbewusst zu handeln.

Abb. 2: Bildungs- und Richtziele des Rahmenlehrplans Geographie, deutsche Fassung General educational aims and targets of Geography in the national curriculum, German version Objectifs généraux et objectifs fondamentaux pour la géographie dans le Plan d'études cadre, version allemande (Schweizerische Konferenz der Kantonalen ERZiehungSdirekToren 1994: 117ff.)

tive legt. Leider gelang es während der Realisierungsphase nicht, einen Kompromiss zwischen den verschiedenen Sichtweisen zu finden, so dass die Erziehungsdirektorenkonferenz schliesslich verschiedenen Versionen ihre Zustimmung gab.

Nach seiner Inkraftsetzung diente der Rahmenlehrplan den Kantonen und Schulen als Grundlage für die neu zu verfassenden kantonalen und schuleigenen Lehrpläne. Doch bevor damit begonnen wurde, änderte der Bundesrat die Maturitätsannerkennungsverordnung (MAV). Die neue Verordnung von 1995 kennt Grundlagen-, Schwerpunkt- und Ergänzungsfächer und gibt Schülerinnen und Schülern mehr Möglichkeiten, aus einem Angebot an Fächern auszuwählen (SCHWEIZERISCHER BUNDESRAT 1995). Geographie ist darin Grundlagen- und Ergänzungsfach. Als Grundlagenfach bildet es zusammen mit Geschichte und Einführung in Wirtschaft und Recht die Fächergruppe "Geistes- und Sozialwissenschaften» (Abb. 3). Mit der Schaffung von
Fächergruppen sollte ein koordinierter und kohärenter Unterricht innerhalb des Bereichs ermöglicht werden, der auf die komplexen Zusammenhänge zwischen Natur, Mensch und Gesellschaft eingeht (SCHWEIZERISCHE MatURITÄtSKommission 1995: 9f.).

Rückblickend kann man sagen, dass die Schaffung des Rahmenlehrplans für das gymnasiale Bildungswesen in der Schweiz von fundamentaler Bedeutung war, denn zum ersten Mal in der fast hundertjährigen Geschichte der Schweizer Gymnasien gibt es damit national verbindliche Ziele darüber, was Gymnasiasten am Ende ihrer Schulzeit wissen und können sollen. Mit der Inkraftsetzung des Rahmenlehrplans war auch die Hoffnung verbunden, dass im Zuge der Neukonzeption der Lehrpläne an den Schulen neue, schülerzentriertere Unterrichtsformen (SCHWEIZERISCHE KONFERENZ DER KANTONALEN ERZIEHUNGSDIREKTOREN 1994: 139), die die kommunikativen Fähigkeiten der Schülerschaft verbessern, sie zu Gruppenarbeit 
anleiten und sie an fächerübergreifendes Denken heranführen, stärker berücksichtigt würden (Maturitäts-Anerkennungsreglement Art. 5; SCHWEIZERISCHER BundesRat 1995).

Nach 1995 begann man in den Schulen mit dem Verfassen der schuleigenen Lehrpläne. Auch dieser Prozess lief basisdemokratisch ab, in dem die Fachschaften ihre Lehrpläne ausgehend von den Voraussetzungen in den Schulhäusern, von den Zielen, Bedürfnissen und Visionen jeder Schule und ihrer Lehrerschaft selber schrieben. Da der Schweizerische Rahmenlehrplan sehr offen gehalten ist, hatten die Lehrkräfte einen grossen Spielraum bei der Zusammenstellung ihrer Unterrichtsprogramme, ihrer spezifischen Ziele, Inhalte, Fertigkeiten und Haltungen, die sie in den Klassen erreichen wollen. Von Seiten der Kantone gab es dafür unterschiedliche Vorgaben, in einigen Kantonen wurden zuvor auch kantonale Rahmenlehrpläne ausgearbeitet.

Obwohl diese Art des Vorgehens ein grosses Mass an Freiheit ermöglichte und man annehmen könnte, dass die Lehrerinnen und Lehrer motiviert an die
Arbeit gingen, waren diese jedoch recht verunsichert, da sie bis anhin keinerlei Erfahrung mit Curriculumsentwicklung hatten und sich überfordert fühlten. Deshalb entwarf die Arbeitsgemeinschaft für Fachdidaktik (AFGg) zusammen mit Lehrerinnen und Lehrern aus allen Landesteilen der Schweiz 1995 einen ModellLehrplan (BALZlı et al. 1995), der den Lehrkräften die Planungsarbeit erleichtern sollte und an alle Mitglieder des Vereins Schweizerischer Geographielehrerinnen und -lehrer (VSGg) verteilt wurde. Er enthält für die Schulgeographie relevante Lernziele und Inhalte und beschreibt auch fachmethodische Kompetenzen, die im Unterricht erreicht werden sollen. Ausserdem erläutert er, wie der zu vermittelnde Stoff in einen regionalen Kontext eingebunden werden kann. Die Fachdidaktiker und Fachdidaktikerinnen wollten mit der Verbreitung des Modell-Lehrplans sicherstellen, dass die Lehrkräfte bei der Konzeption ihrer Lehrpläne von zeitgemässen curricularen Ansätzen, die heute in der Geographiedidaktik üblich sind, ausgehen.

Da nun fast alle Lehrpläne von den Erziehungsdepartementen genehmigt sind - der gesamte Reformprozess wird im Jahr 2001 abgeschlossen sein - stellt

\section{Grundlagenfächer}

\begin{tabular}{|c|c|c|}
\hline 1 & Erstsprache & Alte Sprachen \\
\hline 2 & Eine 2. Landessprache & Moderne Sprache \\
\hline 3 & $\begin{array}{l}\text { Alte Sprachen/Englisch/ } \\
\text { 3. Landessprache }\end{array}$ & $\begin{array}{l}\text { Physik + Anwendungen der } \\
\text { Mathematik }\end{array}$ \\
\hline 4 & Mathematik & Biologie + Chemie \\
\hline 5 & $\begin{array}{l}\text { Naturwissenschaften: } \\
\text { Biologie+Chemie+Physik }\end{array}$ & Wirtschaft+Recht \\
\hline 6 & $\begin{array}{l}\text { Geistes- u. Sozialwissenschaften: } \\
\text { Geschichte + Geographie + } \\
\text { Einführung in Wirtschaft u. Recht }\end{array}$ & $\begin{array}{l}\text { Philosophie + Pädagogische } \\
\text { Psychologie }\end{array}$ \\
\hline 7 & Bildnerisches Gestalten/Musik & $\begin{array}{l}\text { Bildnerisches } \\
\text { Gestalten/Musik }\end{array}$ \\
\hline \multicolumn{3}{|r|}{ 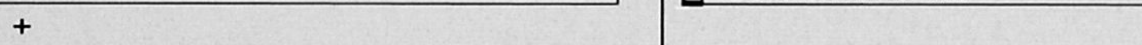 } \\
\hline \multicolumn{3}{|c|}{ Schwerpunktfach } \\
\hline \multicolumn{3}{|c|}{ Ergänzungsfach } \\
\hline \multicolumn{3}{|l|}{+} \\
\hline 10 & Maturaarbeit & \\
\hline 11 & Turnen & \\
\hline
\end{tabular}

\section{Ergänzungsfächer}

Anwendungen d.
Mathematik
Physik
Chemie
Biologie
Geographie
Geschichte
Wirtschaft + Recht
Pädagogik/Psychologie
Philosopie
Religion
Bildnerisch. Gestalten/
Musik
Sport

Abb. 3: Synoptische Darstellung der Maturitätsfächer

The Swiss model curriculum for upper secondary education

Table synoptique des disciplines de maturité à l'échelon suisse

(SCHWEIZERISCHE MaturitätSKommission 1995: 18) 
sich die Frage, ob und wie der Rahmenlehrplan Geographie in den kantonalen und schuleigenen Geographielehrplänen umgesetzt wurde, wie das Fach heute im Gymnasium rezipiert wird, und ob die Empfehlungen des Modell-Lehrplans der AFGg berücksichtigt wurden.

\section{Methoden}

\subsection{Lehrplanauswahl und Analyse}

Diese Studie repräsentiert den Status Quo in der Schweiz in Mai 2000 und basiert auf den Lehr- und Bildungsplänen, die zu diesem Zeitpunkt erhältlich waren. Es ist die erste Studie, die sich seit der Maturitäts- und Lehrplanreform mit Schweizer Geographielehrplänen für die Sekundarstufe II befasst.

Es wurden 26 Lehrpläne untersucht, aus jedem Kanton einer. Von den verwendeten Lehrplänen sind gemäss der Sprachverteilung in den verschiedenen Landesteilen 19 in Deutsch, sechs in Französisch und einer in Italienisch geschrieben.

Die Stichprobenauswahl geschah wie folgt: Die Gesamtpopulation bestand aus den Lehrplänen der 150 Gymnasien, die eidgenössisch anerkannt sind und deren Rektoren Mitglieder der Konferenz Der SchWEIZERISCHEN GyMnasialrekToRen (1999) sind. Von dieser Population sollte für jeden Kanton je ein Lehrplan zufällig ausgewählt werden, um sicherzustellen, dass die Stichprobe nicht einseitig ist und dass alle Regionen und Kulturen vertreten sind.

Bei der Lehrplanauswahl ergaben sich folgende Probleme:

- Fünf Kantone (Waadt, Genf, Wallis - wobei hier nur der frankophone Kantonsteil untersucht wurde-, Graubünden, Kanton Basel-Landschaft) besitzen einen für die Sekundarstufe II gültigen kantonalen Lehrplan und keine schulspezifischen Lehrpläne. Für diese Kantone war die Auswahl der Lehrpläne nach dem Zufälligkeitsprinzip somit hinfällig.

- Der Lehrplan des Kantons Tessin befand sich zum Zeitpunkt der Erhebung noch in der Konzeptionsphase. Ähnlich wie in den frankophonen Kantonen wird es hier einen kantonalen Lehrplan geben, der für alle Maturitätsschulen gültig ist.

- Neun kleinere bis sehr kleine Kantone (Appenzell I.R., Appenzell A.R., Glarus, Jura, Neuenburg, Nidwalden, Obwalden, Schaffhausen und Uri) haben nur je eine bis drei Maturitätsschulen, die bei der Lehrplankonzeption auf Kantonsebene sehr eng zusammengearbeitet haben, so dass auch in diesen Kantonen faktisch nur je ein Lehrplan existiert und keine zufällige Auswahl möglich ist.

- In den verbleibenden 11 Kantonen konnte jede Kantonsschule ihren eigenen Lehrplan schreiben, obwohl in einigen Kantonen (z.B. den Kantonen Aargau, BaselStadt, St. Gallen, Thurgau, Bern) kantonal einheitliche Bildungs- und Richtziele vorgegeben wurden. Aus diesen Kantonen wurden die für die Studie benötigten Lehrpläne mittels einer Zufallstabelle ausgewählt. Drei dieser so bestimmten Schulen stellten ihre Lehrpläne für diese Untersuchung aus verschiedenen Gründen nicht zur Verfügung (keine Antwort von den angefragten Verantwortlichen oder Lehrpläne noch nicht vom Erziehungsrat genehmigt), sodass andere Schulen in diesen Kantonen (es handelte sich um die Kantone FR, BL, TG) um ihre Lehrpläne angefragt wurden. Bezogen auf die Gesamtzahl der Lehrpläne entsprachen diese einem Anteil von 11.5\%.

Die Analyse der 26 Lehrpläne konzentrierte sich auf die folgenden Schlüsselfragen:

a. Stimmen die übergeordneten Ziele in den Lehrplänen mit jenen im Rahmenlehrplan überein?

b. Welcher geographische und pädagogische Ansatz wurde zwecks Konzeption der Lehrpläne gewählt?

c. Welche geographischen Inhalte sollen im Gymnasium unterrichtet, und welche grundlegenden Fertigkeiten und Haltungen sollen erworben werden?

Methodisch wurde eine Kombination von beschreibendem, vergleichendem und analytischem Vorgehen gewählt. Das wichtigste Untersuchungsinstrument in dieser Studie ist der inhaltliche Satz-für-Satz Vergleich der Ziele im Rahmenlehrplan mit jenen in den kantonalen und schuleigenen Lehrplänen. In einem nächsten Schritt erfolgte eine Inhaltsanalyse, um den in den Lehrplänen benutzten geographischen und pädagogischen Ansatz festzustellen, und um herauszufinden, ob und wie die relativ offenen Ziele im Rahmenlehrplan in sehr viel konkretere Grobziele auf Schulebene umgesetzt wurden. Als drittes wurden die in den Lehrplänen formulierten Inhalte, Fertigkeiten und Haltungen separat aufgelistet und zu Kategorien geordnet und interpretiert.

\subsection{Grenzen der Studie}

Diese qualitative Untersuchung beschränkt sich aus ökonomischen und zeitlichen Gründen auf je einen Lehrplan aus den 26 Kantonen, weshalb die Stichprobe nicht als absolut repräsentativ betrachtet werden kann. Mit dem Versuch, die Lehrpläne so weit als möglich zufällig auszuwählen, sollte Einseitigkeit verhindert werden («Purposeful random sampling», siehe GALL et al. 1996: 235).

Ein weiteres Problem lag in den untersuchten Dokumenten selbst. Wie oben erwähnt, gibt es drei Fassungen der Rahmenlehrpläne, die inhaltlich nicht übereinstimmten. Dies machte es schwierig, die gewonnenen Daten zu standardisieren. Da auch die kantonalen und schuleigenen Lehrpläne bezüglich Format, 
pädagogischem Konzept und Detaillierungsgrad enorm variieren, konnte die Datenerhebung nur in einer verkürzten und generalisierten Art und Weise erfolgen. Dadurch ging ein Teil der vorhandenen Information und Authentizität verloren.

\section{Ergebnisse}

\subsection{Vergleich der Ziele}

In nur 13 Lehrplänen sind die dort formulierten Ziele - und zwar sowohl Bildungs- als auch Richtziele - ganz oder überwiegend identisch mit jenen im Schweizerischen Rahmenlehrplan Geographie. In 18 Lehrplänen sind wenigstens die Bildungsziele identisch bis fast identisch, in 14 Lehrplänen sind nur die Richtziele weitgehend identisch. Einige Bildungs- und Richtziele wurden einfach weggelassen, neue wurden hinzugefügt, andere wurden verändert, indem ihnen etwas beigefügt oder indem sie umgeschrieben wurden. So wurden beispielsweise Bildungsziele, die im Rahmenlehrplan stehen, aufgesplittet und erscheinen wieder in detaillierter Form als Richtziele, oder Richtziele wurden zu Bildungszielen umgeschrieben. Dies soll kurz am Beispiel der kantonalen Bildungsziele für das Grundlagenfach Geographie des Kantons Aargau (ErzienungsDEPARTEMENT DES Kantons Aargau 1999) erläutert werden. Alle Zitate sind kursiv gedruckt:

Die Geographie untersucht den Raum in seiner Ganzheit. Dieser Satz kommt so nicht im gesamtschweizerischen Rahmenlehrplan vor. Sie (die Schïlerinnen und Schüler, Verf.) setzen sich mit Veränderungen bewusst auseinander und werden für die Folgen des eigenen Handelns sensibilisiert. Dieses Ziel entspricht sinngemäss den beiden letzten Grundhaltungen, die im Schweizerischen Rahmenlehrplan unter den Richtzielen (Abb. 2) aufgeführt sind. Er (der Geographieunterricht, Verf.) erzeugt Verständnis für Besonderheiten und Probleme anderer Kulturen und Regionen und leistet damit einen Beitrag zur Erziehung, zu Verantwortung und Solidarität gegenüber Mitmenschen. Dieses Bildungsziel geht weiter als jene im eidgenössischen Rahmenlehrplan, wo die Solidarität nicht erwähnt wird.

In anderen Lehrplänen wurden die Ziele zu kompletten Texten verarbeitet, die dann mit dem Titel «Leitidee» in der Art einer Präambel dem Stoffplan vorangestellt sind (z. B. Kantonsschule Kollegium Schwyz 1997).

Viele dieser Eingriffe veränderten die ursprüngliche Bedeutung der Ziele im Rahmenlehrplan. Oft sind sie dadurch inhaltlich kaum wiederzuerkennen und manchmal auch schwerer verständlich.

\subsection{Pädagogischer und geographischer Ansatz}

21 Lehrpläne beruhen heute auf Lernzielen, was einer deutlichen Neuerung entspricht. Die Lernziele begründen, warum ein bestimmtes Thema unterrichtet und welche Fertigkeiten dadurch erreicht werden sollen. Ihr Inhalt ist allgemeingeographisch ausgerichtet (physisch- und humangeographisch). Nur 5 Curricula sind noch in der Form von reinen Themenlisten ohne spezifische Ziele abgefasst.

Was die geographische Ausrichtung betrifft, so benutzten die Hälfte der untersuchten Schulen einen mehr thematischen Ansatz, während die anderen $50 \%$ den thematisch-regionalen Ansatz bevorzugten. Allerdings erwähnten letztere nicht immer die spezifischen Regionen, an denen beispielhaft geographische Konzepte gelernt werden können.

\subsection{Inhalte, Fertigkeiten und Haltungen}

4.3.1 Das grundlegende Geographieverständnis In allen Lehrplänen wird Geographie als eine Raumwissenschaft verstanden. Die übereinstimmende grundlegende Vorstellung ist die, dass die Schülerinnen und Schüler im Geographieunterricht etwas über die räumlichen Wechselbeziehungen zwischen Mensch/ Gesellschaft - Natur/Raum erfahren sollen.

\subsubsection{Geographische Inhalte}

Die Inhalte können in die folgenden Hauptkategorien unterteilt werden: Physische Geographie mit den Teilbereichen Geologie, Geomorphologie, Klimatologie, Ressourcen, Geoökologie und Umweltfragen; Humangeographie mit den Teilbereichen Wirtschafts-, Bevölkerungs-, Siedlungs- und Kulturgeographie (Ethnologie, Kulturlandschaft) sowie Raumplanung. Ganz generell kommt zum Ausdruck, dass $15-50 \%$ der Unterrichtszeit für Themen der physischen Geographie und $50-85 \%$ der Zeit für humangeographische Themen benutzt werden soll. Sehr oft wird aber betont, dass die geographische Raumanalyse im Unterricht ganzheitlich zu erfolgen hat. Im allgemeinen stehen den Lehrkräften für den Geographieunterricht drei Jahre mit durchschnittlich zwei Wochenstunden à 40 bis 45 Minuten zur Verfügung (Abb. 4).

Klassische Themen des Geographieunterrichts wie Astronomie und Kartographie, aber auch Ozeanographie und Bodenkunde, sind häufig in die Lehrpläne des Ergänzungsfaches Geographie verschoben worden. Sie scheinen nicht mehr unabdingbar für ein geographisches Grundverständnis zu sein. Geographie als Ergänzungsfach wird zumeist in Klasse 11 mit durchschnittlich 2.5 Stunden pro Woche und in Klasse 12 mit durchschnittlich 3 Stunden pro Woche angeboten (Abb. 4).

\subsubsection{Regionales Beispiel Europa}

In nur 7 Lehrplänen ist Europa thematisch vertreten, oder es wird zumindest explizit gesagt, dass Europa als regionales Beispiel im Unterricht herangezogen werden soll. 
4.3.4 Kognitive und psychomotorische Kompetenzen Unter den kognitiven Kompetenzen wird hier das Fachwissen und Verständnis für geographische Konzepte verstanden. Wissen und die Fähigkeit zu denken sind Kriterien, welche in allen 26 Lehrplänen im Zentrum stehen und gleich stark betont werden.

Im Gegensatz dazu wird den psychomotorischen und manuellen wie auch technischen Kompetenzen eine geringere Bedeutung beigemessen. Sie kommen aber immerhin in 20 Lehrplänen vor. Man versteht darunter übereinstimmend die Anwendung quantitativer Methoden, die Kartenarbeit, die Analyse von Diagrammen und Profilen, Graphiken, Bildern und Texten, praktische Tätigkeiten im Gelände, gelegentlich auch den Einsatz des Computers für die Anwendung von Simulationen und den Gebrauch des Internet, seltener auch den Gebrauch Geographischer Informationssysteme (GIS) und Globaler Positionierungssysteme (GPS). In 6 Lehrplänen fehlen im Widerspruch zum Rahmenlehrplan Hinweise darauf, dass im Geographieunterricht auch Fertigkeiten gelernt werden.

\subsubsection{Haltungen und soziale Kompetenzen}

Im Rahmenlehrplan der Geographie sind die Grundhaltungen deutlich formuliert. Zusammenfassend können sie wie folgt beschrieben werden: Die Bereitschaft über Werte und Wertesysteme nachzudenken und einen

\section{Geographieunterricht auf der Sekundar- stufe II}

\section{Grundlagenfach}

Klasse $9+10+11$

ca. 80 Unterrichtsstunden pro Jahr

$=$ Total von ca. 240 Stunden

\section{Ergänzungsfach}

Klasse $11+12$

ca. 90 bis 120 Unterrichtsstunden pro Jahr

$=$ Total von ca. 180 bis 240 Stunden

Abb. 4: Klassenstufen mit Geographieunterricht und durchschnittlicher Unterrichtszeit

Annual number of lessons in geography in the 9th-12th grades

Les différents degrés avec l'enseignement de la géographie et le volume d'enseignement moyen verantwortungsbewussten Umgang mit anderen Menschen und der Natur zu pflegen. Ziele, die soziale Kompetenzen betreffen, wie die Fähigkeit zur Kooperation und Kommunikation, fehlen allerdings, obwohl die Maturitäts-Anerkennungsverordnung ausdrücklich soziale Fähigkeiten verlangt (Art. 5, Bildungsziele; SCHWEIZERISCHER BUNDESRAT 1995, SCHWEIZERISCHE MATURITÄTSKOMMISSION 1995).

Erstaunlicherweise sind in nur 12 Lehrplänen Haltungsziele oder solche, die sich auf soziale Kompetenzen beziehen, zu finden. Dieses Resultat ähnelt jenem, das DüRR \& HüLSMANN (1999) in einer Studie über die Lehrpläne 11 deutscher Länder gefunden haben. Nur in zweien wurden soziale Ziele explizit erwähnt. Ähnliches kennt man aus internationalen Studien: Eine Untersuchung über den Geographieunterricht in 31 Ländern, durchgeführt von der IGU Commission on Geographical Education (GERBER 2000: 1ff.), stellt fest, dass Werteerziehung nur von mässiger Bedeutung ist.

\section{Diskussion}

\subsection{Vergleich der Ziele}

Die Maturitäts-Anerkennungsverordnung (MAV) bezieht sich auf den Rahmenlehrplan, auf welchen sich nach dem Willen des Gesetzgebers die Lehrplanentwicklung in den Kantonen abstützen muss (Art. 8, Lehrpläne; SCHWEIZERISCHER BUNDESRAT 1995), damit die Schülerinnen und Schüler während ihrer gymnasialen Ausbildung vergleichbares Wissen und Können erlernen. Um dies zu erreichen, müssen die Ziele in den Geographielehrplänen der Schulen mit jenen im Rahmenlehrplan einigermassen kongruent sein. Die Konzeption des Rahmenlehrplans Geographie ist schlank und offen und ermöglicht viel Gestaltungsspielraum. Dies war auch von seinen Herausgebern, der Erziehungsdirektorenkonferenz, beabsichtigt, von der er als Referenzdokument bezeichnet wird (SCHWEIZERISCHE MatURITÄTSKOMMISSION 1995: 7). Schliesslich kontrollierten am Ende des Reformprozesses die Erziehungsdepartemente der Kantone und, im Falle der eidgenössisch anerkannten Maturitätsschulen, auch die SchweIzerische Maturitätskommission (SMK), die Anerkennungsinstanz des Bundesrates und der Erziehungsdirektorenkonferenz, die schuleigenen Lehrpläne und stellten sicher, dass sie weitgehend mit dem Rahmenlehrplan übereinstimmten.

Es erstaunt daher, dass in nur $13(=50 \%)$ der untersuchten Lehrpläne die Bildungs- und Richtziele mit den gesamtschweizerischen Vorgaben weitgehend identisch sind. Wie kann dieses Resultat erklärt werden? Dazu muss man den Prozess um die Erneuerung der gymnasialen Ausbildung etwas genauer betrachten. 
Die Kantone besitzen die Schulhoheit und regeln die Richtlinien für die gymnasiale Ausbildung selbst. Die alte Maturitäts-Anerkennungsverordnung (SCHWEIZERISCHER BUNDESRAT 1990) setzte aber trotz schmaler Rechtsbasis faktisch die Norm für die allgemeine Hochschulreife und regelte dadurch weit mehr als zur Erreichung des gesetzlichen Zieles notwendig gewesen wäre. «Sie hat dies treuhänderisch gleichsam auch für die Kantone getan, ohne dafür allerdings je einen formellen Auftrag bekommen zu haben» (Zitat: FLAMMER 1996: 63f.). Als anfangs der 90er Jahre die Arbeiten zur Totalrevision der Maturitäts-Anerkennungsverordnung (MAV) aufgenommen wurden, waren sich das Eidgenössische Departement des Inneren (EDI) und die Erziehungsdirektorenkonferenz einig, dass die Frage der Maturitätsanerkennung gemeinsam zu regeln sei. Zu diesem Zweck erarbeiteten Bund und Kantone eine Verwaltungsvereinbarung über die Anerkennung von gymnasialen Maturitätsausweisen (Schweizerischer Bundesrat \& SchweIzerische KONFERENZ DER KaNTONALEN ERZIEHUNGSDIREKTOREN 1995b), die die gemeinsame Regelung der Maturitätsanerkennung enthält (FLAMMER 1996: 64ff.).

Was bedeutet dies nun für den Rahmenlehrplan ausjuristischer Sicht? Im Kommentar zur «Regelung über die Anerkennung von kantonalen Maturitätsausweisen» (SCHWEIZERISCHE MatURITÄTSKOMmISSION 1995:7), welcher sich auf die Anerkennungsregelung der gymnasialen Maturitätsausweise durch Bund und Kantone - das Maturitätsanerkennungsreglement MAR bezieht (SCHWEIZERISCHER BUNdesRat \& SCHWEIZERISCHE Konferenz der Kantonalen ERZIEHUNGSDiReKTOREN 1995a), steht: «Der gesamtschweizerische Rahmenlehrplan (RLP) dient den Kantonen und Schulen als grundsätzliches Referenzdokument für die Ausarbeitung der Lehrpläne. Diese Bestimmung (Art. 8, Maturitätsanerkennungsreglement, Verf.) verleiht dem Rahmenlehrplan zusätzliches Gewicht; seine Aufgabe ist die Koordination der Lehrgänge und Unterrichtsziele der Maturitätsschulen».

Die vorliegenden rechtlichen Quellen beantworten somit die Frage nach der Bedeutung des Rahmenlehrplans klar: Das Maturitätsanerkennungsreglement (MAR) ist formal und inhaltlich für die Kantone verbindlich und stellt die Mindestanforderungen dar. Der Rahmenlehrplan hat jedoch keine Gesetzeskraft, sondern dient der Auslegung des Reglements (MAR). Abweichungen vom Rahmenlehrplan sind möglich, dürfen aber den Sinn und Geist des Reglementes (MAR) nicht beeinträchtigen.

Um weiter zu verstehen, warum die Umsetzung des Rahmenlehrplans im Fach Geographie nur teilweise erfolgt ist, lohnt es sich, auch einen Blick auf den gesamten Reformprozess um die Maturitäts-Anerkennungsverordnung (MAV) zu werfen. Dieser war gekennzeichnet durch eine leidenschaftliche Auseinandersetzung zwischen traditionellen und innovativen Kräften, zwischen Elitismus und «Demokratisierung», zwischen geschlossenem Fächerkanon und offenen Bildungsinhalten (ARNET 1996: 5f.). Es war ein Kampf zwischen jenen, die alte Privilegien verteidigten, jenen, die für soziale Veränderungen eintraten, und jenen, die sich für neue pädagogische Ideen und Tendenzen stark machten:

- Fast 100 Jahre lang - bis zum Beginn der Reform - bedeutete der Begriff «Maturität» ein Privileg, mit welchem der Zugang zu den Schweizer Universitäten verbunden war. Am Ende der 50er Jahre schlossen nur $2-4 \%$ aller 19jährigen ihre Schulbildung mit der Matur ab, 1994 waren es bereits 16\%, 1996 stieg ihr Anteil auf 18\% (Bundesamt Für BILDUNG UND Wissenschaft 1997). Die Expansion war einerseits auf die demographische Entwicklung in der Schweiz zurückzuführen (geburtenstarke Jahrgänge). Andererseits stieg in den 80er Jahren die Nachfrage nach gut ausgebildeten, hochqualifizierten Arbeitskräften, so dass der politische und gesellschaftliche Druck auf die gymnasiale Ausbildung in Richtung einer Öffnung wuchs (MeYLAN 1996: 9). Viele traditionsreiche und prestigeträchtige Kantonsschulen wollten jedoch keine Veränderung des Fächerkanons. Die Reform wurde gleichgesetzt mit dem Schlagwort «Niveauverlust» (MEYLAN 1996: 32), welcher sich schwerwiegend auf die Selektion auswirke.

- Ein zweiter Grund bestand darin, dass Gymnasiallehrerinnen und -lehrer in der Schweiz neben ihrer Lehrerausbildung ein abgeschlossenes Fachstudium hinter sich haben. Einige erwerben sogar einen Doktortitel. Sie betrachteten sich daher eher als Fachexperten, die ohne «Weisungen von oben» unterrichten, und nicht als «blosse» Erzieher (MEYLAN 1996: 29).

- Ein weiterer Grund ist darin zu suchen, dass sich die Kantone nicht unter eidgenössische Qualitätskontrolle begeben wollten. Mehr als 150 Jahre lang bestimmten die Kantone alle mit der Schulbildung verbundenen Angelegenheiten selbst und liessen sich von der Eidgenossenschaft nicht hineinreden. Folglich ist das Schweizerische Bildungswesen sehr komplex strukturiert und räumlich fragmentiert. Es spiegelt die grundlegende Schweizer Überzeugung, dass schulische Ausbildung am besten auf lokaler und regionaler Ebene kontrolliert werden kann. Der Bundesregierung fehlen eine ausreichend politische Legitimation und die administrativen Ressourcen, um einen bedeutenden Einfluss auf die Kantone auszuüben und als Partner der Kantone in der Bildungspolitik aufzutreten (HEGA 2000: 29).

- Ein vierter Grund liegt in der Tatsache, dass in der Schweiz die Gymnasialpolitik der Hochschulpolitik unterstellt ist. Das Maturitätszeugnis berechtigt die Maturandinnen und Maturanden zum prüfungsfreien 
Übertritt in eine Universität. Auch die Universitäten befürchteten, dass mit der Maturitätsreform ein Niveauverlust der gymnasialen Ausbildung verbunden sei und die zukünftigen Studierenden nicht mehr genügend selektioniert würden.

Aus diesen Gründen bestand wenig Enthusiasmus, Bereitschaft und Eifer, die Reform auf kantonaler Ebene umzusetzen (MeYlan 1996: 37). Davon betroffen war im übrigen nicht nur das Fach Geographie. Es wäre deshalb interessant, auch die anderen Maturitätsfächer einer Analyse zu unterziehen.

Die Schnittmenge derjenen Bildungs- und Richtziele des Rahmenlehrplans, die in allen 26 untersuchten Lehrplänen identisch sind, ist die Menge der Basisziele, die im Geographieunterricht an Schweizer Kantonsschulen übereinstimmend verfolgt werden. Sie gibt auch den Universitäten an, was sie von den Studierenden bei Studienbeginn im Minimum erwarten können. Wegen der inhaltlichen Unterschiede in den drei Fassungen des Rahmenlehrplans wurden sie nur für die deutsche Version bestimmt. Dies geschah, indem alle jene Bildungs- und Richtziele, die in den schuleigenen Lehrplänen stark verändert worden waren oder gar fehlten, aus dem Rahmenlehrplan gestrichen wurden. Abb. 5 zeigt diese Schnittmenge.

Diese übereinstimmenden Basisziele demonstrieren ein interessantes Phänomen: Auch wenn der Lehrplan jeder einzelnen Schule anders aussieht, zeigt die Abbildung 5 deutlich, dass nach dem oben beschriebenen Vorgehen die Hälfte der Bildungsziele aus dem Rahmenlehrplan verschwunden ist. Übrig blieben jene, die am ehesten der heutigen Definition des Schulfaches Geographie entsprechen. Die Richtziele als solche sind noch gut erkennbar, wurden aber stark verkürzt, besonders im Bereich der Fertigkeiten.

\subsection{Pädagogischer und geographischer Ansatz}

21 Lehrpläne basieren auf Lernzielen, 5 sind reine Themenlisten. Dieses Ergebnis zeigt klar, dass die Auseinandersetzung mit der Lehrplanreform zu einer neuen Art des Denkens über Lehrpläne geführt hat. In den curricularen Prozess, der von vier grundlegenden Einheiten ausgehen sollte - den Lernzielen, den Inhalten, der Lernerfahrung/dem Unterricht und der Evaluation (Graves 1996: 74) -, bezog man hier immerhin die beiden ersten Einheiten ein. Auch über die im Unterricht anzuwendenden Methoden werden in einigen Lehrplänen Angaben gemacht, während über die Evaluation keine Aussagen vorliegen. Dies ist auch in den gesetzlichen Grundlagen nicht vorgesehen. In jenen Schulen, deren Lehrpläne nur Themenlisten sind, scheint dieser Entwicklungsprozess nicht stattgefunden zu haben. Die Ursachen hierfür sind unbekannt. Es ist aber bedauerlich, dass hier eine Chance verpasst wurde, den formalen Rahmen des Geographieunterrichts neu zu definieren und dabei über die eigenen aktuellen und zukünftigen Fachziele und die Ziele der Schule zu reflektieren.

Von den geographischen Ansätzen wurden der thematische und der thematisch-regionale gleichermassen berücksichtigt (50\%). Auch dieses Ergebnis zeigt, dass sich der Geographieunterricht in der Schweiz in den letzten 20 Jahren verändert hat, denn in den alten Lehrplänen aus den 80er Jahren ist die Geographie noch sehr stark deskriptiv-schematisch, ganz auf das länderkundliche Schema ausgerichtet. Das Ergebnis macht aber auch deutlich, dass der alte Dualismus der geographischen Wissenschaften zwischen Thematischer und Regionaler Geographie noch nicht überwunden ist. Nach HaUbrich et al. (1997: 152f.) folgt der thematische Ansatz der sachlich-thematischen Kontinuität. Unter Anwendung exemplarischer Verfahren konzentriert er sich auf den Erwerb von nomothetischen Kategorien. Der thematisch-regionale Ansatz stützt sich auf eine thematisch ausgerichtete Geographie, die in einen regionalen Rahmen eingebettet ist. Da die Thematische und Regionale Geographie als komplementär zu betrachten sind, hat sich der thematisch-regionale Ansatz heute beispielsweise in vielen deutschen Lehrplänen durchgesetzt. Es ist daher bedauerlich, dass dieses, auch im Modell-Lehrplan der AFGg (BalzLI et al. 1995) empfohlene Konzept keine grössere Akzeptanz gefunden hat. Schliesslich bestand das Ziel des Modell-Lehrplans in Anlehnung an HaUBrich et al. (1997: 154) darin, den Lehrkräften zu vermitteln, dass geographische Inhalte in regionalem Kontext unterrichtet werden sollen, um geographisches Wissen immer mit einem regionalen Rahmen verknüpfen zu können.

\subsection{Inhalte, Fertigkeiten und Haltungen}

\subsubsection{Das grundlegende Geographieverständnis}

Das Verständnis der Geographie als Raumwissenschaft deckt sich in allen untersuchten Lehrplänen mit den Zielen im Rahmenlehrplan, der die räumliche Orientierung, das Verständnis für natürliche und anthropogen-induzierte räumliche Prozesse und verantwortungsvolles Raumverhalten ausdrücklich erwähnt.

\subsubsection{Geographische Inhalte}

Die Inhaltsanalyse der Lehrpläne zeigt den Wandel, der sich in der Perzeption der Schulgeographie vollzogen hat: Heute wird der Humangeographie eine weit grössere Bedeutung beigemessen. Dies deckt sich auch mit den Anforderungen des neuen Maturitätsanerkennungsreglements (MAR), in welchem die Geographie, zusammen mit Geschichte und Einführung in Wirtschaft und Recht. Teil des Fachbereichs Geistes- und Sozialwissenschaften ist. Innerhalb der Humangeographie ist die Idee der «Inwertsetzung 


\section{Der Rahmenlehrplan Geographie für die Maturitätsschulen}

\section{Allgemeine Bildungsziele}

Das Zusammenwirken und die gegenseitige Beeinflussung von Mensch und Natur soll ... im Geographieunterricht (Verf.) ... verständlich werden.

Die Geographie enthält Elemente des natur- und humanwissenschaftlichen Denkens; deshalb verbindet sie die beiden Bereiche. Sie fördert das Erkennen von Zusammenhängen und regt die fächerübergreifende Behandlung von Themen an.

\section{Richtziele}

\section{Grundkenntnisse}

- Die fachspezifischen Grundbegriffe kennen.

- Über ein topographisches Grundwissen verfügen, um aktuelle Ereignisse geographisch deuten und einordnen zu können.

- Landschaftselemente und ihre raumprägenden Faktoren kennen.

- Die Prozesse des Landschaftswandels erfassen.

\section{Grundfertigkeiten}

- Karten lesen und sich im Gelände zurechtfinden.

- Geographische Darstellungsmethoden anwenden, thematische Karten, Profile, Diagramme, Statistiken, Modelle, Bilder und Texte interpretieren und z.T. selbst entwerfen; Ergebnisse geographischer Untersuchungen verständlich darstellen und weitergeben.

\section{Grundhaltungen}

- Die Begegnung mit anderen Menschen, Kulturen und Landschaften als Bereicherung erfahren und durch die Vergleiche die eigene Umwelt besser verstehen.

- Durch persönliche Erlebnisse und Erfahrungen seine Einstellung überdenken, sich auftauchender Probleme bewusst werden und sich für deren Lösung einsetzen.

Abb. 5: Übereinstimmende Basisziele zwischen dem Rahmenlehrplan und 15 Lehrplänen Deutschschweizer Kantonsschulen

Matching core content of the objectives in the national curriculum and those in 15 school-based curricula from schools in German speaking cantons

Objectifs fondamentaux coïncidant avec le Plan d'études cadre et quinze plans d'études d'écoles suisses alémaniques

eines Raumes», die noch in den alten Lehrplänen stark mit der Länderkunde verknüpft war, verschwunden. Die neue Humangeographie stellt den ökologischen Ansatz unter Betonung der Nachhaltigkeit ins Zentrum. Dieses Vorgehen wurde von den Fachdidaktikern auch im Modell-Lehrplan empfohlen und offensichtlich übernommen.

\subsubsection{Regionales Beispiel Europa}

In den kantonalen und schuleigenen Lehrplänen für die Sekundarstufe II ist die Festschreibung des Themas
Europa erstaunlich gering; nur 7 Lehrpläne verlangen dies. Der Grund hierfür könnte einerseits sein, dass Europa Thema des Geographieunterrichts auf der Sekundarstufe I ist. Dort wird es in der Art und Weise unterrichtet, dass für diese Altersstufe interessante Teilthemen wie «Vulkanismus und Erdbeben in Italien», «Küstenformen in Frankreich», «Gletscherspuren in Skandinavien», «Steinkohlevorkommen im Ruhrgebiet» usw. exemplarisch behandelt werden. Möglicherweise war dies ein Grund, warum Europa in vielen Lehrplänen der Sekundarstufe II nicht mehr the- 
matisiert wird. Es erscheint aber unwahrscheinlich, dass Europa, insbesondere die wirtschaftlichen Beziehungen zwischen Europa und der Schweiz, im Geographieunterricht der gymnasialen Oberstufe nicht mehr vorkommen. In den thematisch angelegten Lehrplänen wird dies jedoch nicht sichtbar, da der regionale Bezug fehlt. Deshalb muss dieser Frage in einer weiterführenden Untersuchung nachgegangen werden.

Obwohl die Schweiz nicht Mitglied der Europäischen Union ist, wird das Thema Europa mit fortschreitender europäischer Integration auch für die Schweiz immer wichtiger. Auch wenn unser Land nicht Teil eines vereinigten Europas sein möchte, sollten unsere Lehrpläne sicherstellen, dass die Schülerinnen und Schüler das vereinigte Europa als politisches, wirtschaftliches und kulturelles Gebilde wahrnehmen und seine Schaffung als eine zukünftige Herausforderung akzeptieren. Gemäss Haubrich et al. (1997: 128) muss man nicht begeistert von Europa sein, aber über den Kontinent und die aktuellen Vorgänge informiert sein. Auch sollten junge Menschen sich ermutigt fühlen, aktiv, optimistisch und phantasievoll an der Kooperation mit Europa teilzunehmen. Wenn man die neuen Geographielehrpläne wortwörtlich auslegt, kann bezweifelt werden, ob das Fach diesem Anspruch gerecht werden kann.

Dafür springen andere Maturitätsfächer in diese Lücke, obwohl dies in den Zielen im Schweizerischen Rahmenlehrplan so nicht vorgesehen ist. Das Fach Wirtschaft und Recht diskutiert beispielsweise den "Aufbau und die Ziele der Europäischen Union» (Kantonsschule Obwalden 1997: 43). Das Thema soll interdisziplinär mit der Geschichte angegangen werden. Auch die Sprachen nehmen sich des Themas Europa an. So wird im Fach Italienisch die Geographie Italiens (KaNTONALER LEHRPLAN FÜR DIE THURGAUISCHEN MATURITÄTSSCHULEN 1996: 25, KantonsSCHULE Glarus 1996: 15), im Französisch die Landeskunde Frankreichs (Kantonsschule Glarus 1996: 13) und im Englisch die Landeskunde der angelsächsischen Länder durchgenommen (KANTONALER LEHRPLAN FÜR DIE THURGaUisCHEN MatURITÄTSSCHUlen 1996: 29). Es wird offensichtlich, dass sich die Geographie in Zukunft um eine fächerübergreifende Zusammenarbeit bemühen muss, will sie nicht mit der Zeit auf der Sekundarstufe II den Einfluss auf ein ihr ureigenes Teilgebiet, die Regionale Geographie Europas, verlieren.

\subsubsection{Kognitive und psychomotorische Kompetenzen} Obwohl der Rahmenlehrplan gleichermassen kognitive wie auch psychomotorische Kompetenzen betont, liegt der Schwerpunkt in allen 26 untersuchten Lehrplänen auf dem Erwerb von Fachwissen. Dies kann mit der Tradition der Kantonsschulen, vor allem Fach- wissen zu vermitteln, begründet werden. Sie betrachteten sich seit jeher als Institutionen mit einem wissenschaftspropädeutischen Anspruch, die auf die Universitäten vorbereiten. Dies spiegelt sich auch in der Gymnasiallehrerausbildung, in der das Fachstudium, das mit einem Lizenziat oder Diplom abgeschlossen wird und inhaltlich kaum auf den Schulstoff ausgerichtet ist, der zentrale Ausbildungsteil ist und sicherstellt, dass die Lehrkraft in ihrem Fachgebiet wissenschaftlich kompetent ist.

Was die psychomotorischen Kompetenzen angeht, so sieht man, dass sich die Vorstellungen von Klassenunterricht stark verändert haben. In 20 Lehrplänen bedeutet Geographieunterricht nicht mehr nur lehrerzentriertes Unterrichten und das Reproduzieren von Fakten. Die Idee, dass man lernt, indem man etwas tut (learning by doing), hat sich durchgesetzt: Eine geographisch gebildete Person besitzt nicht nur Fachwissen, sondern weiss auch, wie man geographische Methoden anwendet. Dies verlangt auch das Maturitätsanerkennungsreglement in Art. 5 (SCHWEIZERISCHE MATURITÄTSKOMMISION 1995). Dass in noch 6 Lehrplänen keine Fertigkeiten ausgewiesen werden, wird so interpretiert, dass sich in diesen Fällen die Autorenschaft zu wenig bewusst darüber war, dass Lernen auf verschiedenen Ebenen erfolgt und dass Fertigkeiten nur dann erworben werden können, wenn man den Schülerinnen und Schülern Lernerfahrungen ermöglicht, die im voraus mit dem Inhalt abgestimmt werden müssen.

\subsubsection{Haltungen und soziale Kompetenzen}

In unserem Zeitalter der Globalisierung ist es notwendig, nicht nur Fakten über andere Kulturen zu kennen, sondern auch die Lebensweisen in anderen Kulturen zu verstehen, kritisch über Wertesysteme nachzudenken und soziale Kompetenzen im Klassenzimmer zu entwickeln. Dies sind einige Grundlagen, um friedlich in einer multikulturellen und demokratischen Gesellschaft zusammenzuleben. Trotzdem richtet sich in nur 12 Lehrplänen der Blick auf Grundhaltungen und soziale Kompetenzen, obwohl das Fach Geographie unzählige Gelegenheiten bietet, um über Kulturen und ethische Werte nachzudenken und Kommunikation und Kooperation zu praktizieren. Offensichtlich hielten es die Schulen für ausreichend, dass Grundhaltungen im Rahmenlehrplan formuliert sind, taten sich aber schwer damit, diese auf der Ebene der schuleigenen Lehrpläne zu spezifizieren.

Dieses Ergebnis deckt noch ein weiteres Problem der Maturitätsreform auf. Der Rahmenlehrplan geht auf das Jahr 1994 zurück, während die Diskussion um offene Unterrichtsformen und ihre positiven Auswirkungen auf soziale Kompetenzen erst nach deren Inkraftsetzung begann. Somit wurde eine Chance 
vertan, eine breitere Sicht der sozialen Kompetenzen nicht nur die sogenannten Grundhaltungen - im Rahmenlehrplan festzuschreiben und eine grössere Vielfalt an Unterrichtsmethoden im Schulalltag zu verlangen. Trotzdem werden heute in den Klassenzimmern der gymnasialen Oberstufe vielfältige Unterrichtsmethoden, wie Projektarbeit, Leitprogramme, Fallstudien, entdeckendes Lernen, Rollenspiele und Gruppendiskussionen praktiziert (REINFRIED 2000). Dieses Ergebnis bestätigt die Feststellung von KüNZLI \& SANTINTI-AMGARTEN (1999:146), dass eine Lehrplanreform nicht nur darauf abzielt, den zukünftigen Unterricht zu verändern, sondern auch bereits existierende Unterrichtspraxis und Innovationen festschreibt.

Dieser Punkt der Untersuchung zeigt ausserdem, dass zu dem Zeitpunkt, als die Reform begann, kein ganzheitliches Konzept über die Lehrplanentwicklung und Maturitätsreform existierte. Heute legt der Rahmenlehrplan klar fest, was die Schülerinnen und Schüler im Fach Geographie wissen und können sollten. Er garantiert jedoch nicht, dass der Geographieunterricht besser wird, weil die vier Grundpfeiler des curricularen Prozesses, die Ziele, die Inhalte, der Unterricht und die Evaluation (Graves 1996: 74) nicht gleichzeitig diskutiert wurden. Heute weiss niemand, wie es um die Qualität der gymnasialen Ausbildung in den verschiedenen Kantonen wirklich steht und ob nicht gemeinsame, vergleichbare, auf den Rahmenlehrplan abgestützte Prüfungsnormen sinnvoll wären, um die Leistungen der Schülerinnen und Schüler wirksam gesamtschweizerisch zu vereinheitlichen (MEYLAN 1996:37). Die Idee, die Unterrichtsqualität im Gymnasium auf der Basis der Ziele im Rahmenlehrplan zu evaluieren, ist heute noch kein Thema in der Schweiz. Aus ökonomischen und politischen Gründen erscheint es auch unrealistisch, dass dies in naher Zukunft realisiert werden könnte.

\section{Schlussfolgerungen}

In den letzten 10 Jahren erlebten die Schweizer Gymnasien eine Lehrplanreform, die trotz beträchtlichem Widerstand in den Kantonen umgesetzt wurde und heute eine nationale Realität ist. Gesamthaft betrachtet hat sich die Perzeption des Schulfaches Geographie auf der Sekundarstufe II grundlegend verändert. Das Fach unterrichtet heute zeitgemässe Inhalte und will neben Wissen auch Methodenkompetenz vermitteln. Die Erziehung zu Haltungen und Werten wird dagegen in vielen Geographielehrplänen noch vernachlässigt. Insgesamt wird die Reform einen positiven Einfluss auf den Geographieunterricht im 21. Jahrhundert haben. Es dürfte interessant sein, in einem nächsten Schritt zu untersuchen, was wirklich im Geographieunterricht geschieht und wie Geographielehrerinnnen und -leh- rer ihre Lehrpläne in das tägliche Unterrichtsgeschehen umsetzen.

\section{Literatur}

ARnet, M. (1996): Einleitung. - In: EDK: Von der «Mittelschule von morgen» zur Maturitätsreform. Studien und Berichte 8, Bern: 5-6.

Balzli, M., Collet, G., Gilgen, B., Hasler, M., Heim, H., Hertig, P., Theuriat, P.-Y., Reinfried, S., Speck, P. \& H.R. VOLKarT (1995): Modell-Lehrplan Sekundarstufe II. - AFGg-Dokument 4, Zentralstelle für die Weiterbildung der Mittelschullehrer. - Luzern: WBZ. BUNDESAMT FÜR BILDUNG UND WISSENSChAFT (1997): Bildungsstatistik 1996. - Pressemitteilung vom 19.9.97. www.statistik.admin.ch/news/archiv97/

CONFÉRENCE SUISSE DES DIRECTEURS CANTONEAUX DE L'INSTRuCtion Publique CDIP (1994): Plan d'étude cadre pour les écoles de maturité. $-=$ Dossier CDIP 30B, Bern: CDIP.

CONFERENZA SVIZZERA DEI DIRETTORI CANTONALI DELLA pubBlica eduCAZIONE CDPE (1994): Piano quadro degli studi per le Scuole svizzere di maturità $-=$ Fascicolo CDPE 30C, Bern: CDPE.

DürR, H. \& M. HülsmanN (1999): Basisinhalte des Erdkundeunterrichts. - In: Praxis Geographie 7/8: 61-62. ERZIEHUNGSDEPARTEMENT des Kantons Aargau (1999): Bildungs- und Richtziele des Grundlagenfaches Geographie. Genehmigt vom Erziehungsrat am 23.9.1999, Aarau.

Flammer, E. (1996): Neue Trägerschaft für die Maturitätsanerkennung.- In:EDK:Von der «Mittelschule von morgen» zur Maturitätsreform. Studien und Berichte 8, Bern: 61-71.

Gall, M.G., Borg, W. R. \& J.P. Gall (1996): Educational Research. - New York: Longman.

Gerber, R. (2000): The State of Geographical Education. - In: Countries Around The World. IGU-CGE Kyongju Symposium, Korea, Proceedings: 1-5.

Graves, N. (1996): Curriculum development in geography: An ongoing process. - In: KENT, A. et al.: Geography in Education. Viewpoints on Teaching and Learning, Cambridge: 72-99.

Haubrich, H., Kirchberg, G., Brucker, A., Engelhard, K., Hausmann, W. \& D. Richter (1997): Didaktik der Geographie Konkret. - München: Oldenbourgh.

HeGA, G.M. (2000): Federalism, Subsidiarity and Education Policy in Switzerland. - In: Regional \& Federal Studies,10 (1), London: 1-35.

KaNTONALER LeHRPLAN Für Die THURgauischen MatuRITÄTSSCHULEN (1996): LZM 96 (prov. Fassung). - Frauenfeld, $119 \mathrm{~S}$.

Kantonsschule Glarus (1996): Lehrplan für das Gymnasium. - Glarus, 97 S.

KantonsSChUle Kollegium SCHWyz (1997): Lehrplan Geographie. - Schwyz: 68-74. 
Kantonsschule Obwalden (1997): Lehrpläne MAR 95. - Sarnen, $128 \mathrm{~S}$.

KONFERENZ SCHWEIZERISCHER GyMNASIALREKTOREN (1999): Mitgliederliste vom 30. August 1999.

Künzli, R. \& B. Santinti-Amgarten (1999): Wie Lehrpläne umgesetzt werden. - In: KüNZLI, R. et al.: Lehrplanarbeit. NFP 33, Zürich: 144-167.

Meylan, J.-P. (1996): Die Erneuerung des Gymnasiums und die Anerkennung der Maturitäten - Stationen der Debatte 1968-1995. - In: EDK: Von der «Mittelschule von morgen» zur Maturitätsreform. Studien und Berichte 8, Bern: 7-45.

Reinfried, S. (2000): The Swiss Curriculum Reform and its Impact on High School Geography - A Curricular Change that Promotes Lifelong Learning. $29^{\text {th }}$ International Geographical Congress, Abstracts, Seoul: 444-445.

SCHWEIZERISCHE Konferenz Der Kantonalen ErzieHUNGSDIREKTOREN EDK (1994): Rahmenlehrplan für die Maturitätsschulen. - = Dossier EDK 30A. EDK: Bern.

SCHWEIZERISCHE MaturitätSKOMMISSION (1995): Regelung über die Anerkennung von kantonalen Maturitätsausweisen. Kommentar. - Bern: SMK

SCHWEIZERISCHER BUNDESRAT (1990):Verordnung über die eidgenössischen Maturitätsprüfungen 413.12./Stand am 1. April 1990, Bern.

SCHWEIZERISCHER BundeSRAT (1995): Verordnung über die Anerkennung von gymnasialen Maturitätsausweisen (Maturitäts-Anerkennungsverordnung MAV) vom 15. Februar 1995, Bern. - 1. August 1995, Bern.

SCHWEIZERISCHER BundesRat \& SChWEIZERISCHE KoNFERENZ DER KANTONALEN ERZIEHUNGSDIREKTOREN (EDK) (1995a): Verordnung des Bundesrates/Reglement der EDK über die Anerkennung von gymnasialen Maturitätsausweisen (MAR) vom 16. Januar/15. Februar 1995. - 1. August 1995, Bern.

SChWEIZERISCher Bundesrat \& SChweizerische KonFERENZ DER KANTONALEN ERZIEHUNGSDIREKTOREN (EDK) (1995b): Verwaltungsvereinbarung über die Anerkennung von gymnasialen Maturitätsausweisen. BBI 1995-1. - 1. August 1995, Bern.

Tschanz, M. (1997): Der VSGg 1988-1992: Der lange Weg zum Rahmenlehrplan. - In: EgLI, H.-R. et al. (Hrsg.): Spuren, Wege und Verkehr. Festschrift für Klaus Aerni. Jahrbuch der Geographischen Gesellschaft 60, Bern: 39-40.

Eine Liste mit den für die Studie benutzten Lehrplänen kann bei der Autorin bezogen werden.

\section{Zusammenfassung: Geographieunterricht in Schweizer Gymnasien nach der Maturitätsreform - Eine Analyse der neuen Geographielehrpläne}

Die Studie beschreibt die Ergebnisse der Lehrplanreform im Fach Geographie, die in den letzten 10 Jahren in den Schweizer Gymnasien stattfand. Seit 1994 gibt es erstmals in der Geschichte der Maturitätsschulen einen für die ganze Schweiz verbindlichen Rahmenlehrplan für die Ausbildung auf der Sekundarstufe II. Die in ihm formulierten Bildungs- und Richtziele dienten als Grundlage für die Konzeption der kantonalen und schuleigenen Lehrpläne. Um herauszufinden, wie das Fach Geographie heute in den Schulen wahrgenommen wird, werden 26 Lehrpläne mit dem Rahmenlehrplan verglichen. Es hat sich herausgestellt, dass nur die Bildungs- und Richtziele von 13 Lehrplänen mit jenem im Rahmenlehrplan Geographie übereinstimmen. Dieser Befund kann nur auf dem Hintergrund der politischen Situation in der Bildungslandschaft der Schweiz verstanden werden. Trotzdem hat sich die Schulgeographie auf der Sekundarstufe II zu einem modernen Unterrichtsfach gewandelt, das nicht nur den Erwerb von Wissen, sondern auch von Fertigkeiten betont, gleichzeitig aber in vielen Fällen die affektiven Kompetenzen vernachlässigt.

Summary: Teaching Geography in Swiss High Schools after the School Certificate Reform - an analysis of new Geography curricula

This article describes the results of a curriculum reform in Geography in Swiss upper secondary schools which took place during the last ten years. In 1994, for the first time in Swiss school history, a federally accepted national curriculum for upper secondary education was introduced. The aims and objectives defined therein served as the basis for the conceptualization of individual school curricula. In order to evaluate the acceptance of the national curriculum of geography and the subject as a whole the author compared school-based curricula with the national curriculum. The results show that in only $50 \%$ of the school-based curricula, the objectives correspond more or less with those in the national curriculum. This can only be interpreted in the broader context of the Swiss educational system and its present-day political situation. Nevertheless, Geography in Swiss upper secondary schools has been transformed into a school subject with modern content, emphasizing not only the acquisition of knowledge but also skills. Social skills in particular had previously been neglected in many curricula.

Résumé: L'enseignement de la géographie dans les gymnases suisses après la réforme de la maturité - une analyse des plans d'études en géographie

Cette recherche présente les résultats de la réforme du plan d'études (qui a été initiée il y a une dizaine d'années dans les gymnases suisses) au niveau de la géographie. En effet, depuis 1994 et pour la première fois dans l'histoire des écoles de maturité, l'enseignement au deuxième degré secondaire doit se conformer à un Plan d'études cadre, obligatoire dans toute la Suisse. Les objectifs généraux et fon- 
damentaux qui y figurent ont servi de base à la conception des plans d'études cantonaux et spécifiques à chaque établissement scolaire. Pour découvrir comment la géographie était perçue aujourd'hui dans les écoles, l'auteur a comparé 26 plans d'études avec le Plan d'études cadre. Il en est ressorti que 13 plans d'études seulement avaient fixé des objectifs généraux et fondamentaux coïncidant avec ceux du Plan d'études cadre. La situation politique dans le paysage suisse de la formation en est la seule explication. Malgré tout, la géographie enseignée au deuxième degré secondaire s'est transformée en une discipline moderne, qui met l'accent non seulement sur l'acquisition de connaissances, mais aussi d'aptitudes générales. Cependant, en même temps, elle néglige encore très souvent les compétences affectives.

Dr. sc. nat. Sibylle Reinfried, Universität Zürich, Abteilung für das Höhere Lehramt Mittelschulen, Winterthurerstrasse 30, CH-8033 Zürich 6.

e-mail: s.reinfried@active.ch 